\title{
Sound scattering by live zooplankton and micronekton: Empirical studies with a dual-beam acoustical system
}

\author{
Peter H. Wiebe \\ Woods Hole Oceanographic Institution, Woods Hole, Massachusetts 02543 \\ Charles H. Greene \\ Ocean Resources and Ecosystems Program, Corson Hall, Cornell University, Ithaca, New York 14853 \\ Timothy K. Stanton \\ Woods Hole Oceanographic Institution, Woods Hole, Massachusetts 02543 \\ Janusz Burczynski \\ BioSonics, Incorporated, Seattle, Washington 98105
}

(Received 20 April 1990; accepted for publication 11 July 1990)

Measurements and analyses are presented of the backscattering of $420-\mathrm{kHz}$ sound by 43 individual animals of representative zooplanktonic and micronektonic taxa. Direct measurements of an individual's target strength were made with a commercial dual-beam sonar system in an enclosure filled with filtered seawater deployed off a dock at Friday Harbor, Washington. The dependence of target stengths upon individual length, wet weight, and dry weight was investigated. In addition, the target strength and statistical variations of echo amplitude due to variations in shape and orientation of the organism were compared with acoustic scattering models involving different shapes (the general shapes of the sphere, and straight and uniformly bent finite cylinders were used along with attempts to take into account roughness). It was found that: (1) backscattering cross sections are proportional to volume of the organisms rather than area as would be predicted by a sphere scattering model, (2) mean target strength based on average backscattering cross section is best described by the bent cylinder model whose modal series solution is truncated, and (3) the fluctuations of the echo amplitudes are well described by the Rice probability density function whose shape parameter is related to the randomly rough straight cylinder model. These extensive studies showed conclusively that the elongated animals scattered sound more like elongated targets than spherical ones, thus demonstrating the need for models more sophisticated than the spherical ones routinely used to date. The data and model analyses provide a basis for devising future acoustical data acquisition and processing techniques for bioacoustical field studies.

PACS numbers: 43.30.Ft, 43.30.Pc, 43.30.Xm

\section{INTRODUCTION}

At acoustical frequencies above $1 \mathrm{kHz}$, a large proportion of oceanic volume reverberation is biological in origin (Farquhar, 1971; Anderson and Zahuranec, 1977; Clay and Medwin, 1977). A significant fraction of this reverberation is associated with large zooplankton and micronekton (Sameoto, 1976; Macaulay, 1978; Pieper, 1979; Greenlaw, 1979; Holliday, 1980; Holliday and Pieper, 1980; Pieper and Holliday, 1984; Falk-Petersen and Kristensen, 1985; Kristensen and Dalen, 1986; Greene et al., 1988; Greene et al., 1989a). Despite the importance of these organisms in scattering underwater sound, little empirical data are available on their individual sound-scattering properties (but see Greenlaw, 1977; Richter, 1985a). Furthermore, what little data are available have been collected almost exclusively from preserved specimens. Since preservation has been demonstrated to have a major effect on the physical and acoustical properties of zooplankton and microekton (Greenlaw, 1977; Greenlaw and Johnson, 1983; Richter, 1985a), such data should be used cautiously in testing theoretical sound- scattering models and interpreting the results from acoustical field studies. This latter point is of increasing practical relevance as field studies employing acoustical methods to determine animal distributions in the ocean become more widespread.

Over the past decade, ocean scientists have witnessed the rapid development of two acoustical methods for estimating the biomass, numerical abundance and size distribution of zooplankton and micronekton (see reviews by Greene et al., 1989a; Greene and Wiebe, 1990). The first of the methods to be developed has involved the use of inversion techniques to estimate acoustical size distributions from multiple-frequency volume-backscattering data. Since the beginning of the 1980's, several groups of investigators have used these inversion techniques to apportion estimates of zooplankton and micronekton biomass concentration into different acoustical size classes (Holliday, 1980; Pieper and Holliday, 1984; Falk-Petersen and Kristensen, 1985; Costello et al., 1989; Holliday et al., 1989). Subsequent to their efforts, first Richter (1985a,b) and then our group (Greene 
and Wiebe, 1988; Greene et al., 1988, 1989a,b) have chosen to adopt a second simpler method, developing an approach that involves use of the dual-beam technique.

The dual-beam technique was developed in the 1970's for survey applications in fisheries research (see review by Ehrenberg, 1989). Subsequent refinement of the technique has made it feasible for investigators to resolve and analyze echoes returning from individual animals as small as zooplankton (Richter, 1985a; Greene et al., 1989a). This capability has made it possible to directly estimate in situ the acoustical size distribution of a zooplankton and micronekton assemblage. When the results of such an analysis are combined with the results from an echo integration analysis of the corresponding volume-backscattering data, estimates of numerical abundance and biomass concentration can be apportioned into different acoustical size classes (Greene $e t$ al., 1989a; Greene and Wiebe, 1990).

In this article, we present results from a more extensive analysis of experimental data collected in 1987 and reported upon previously (Greene et al., 1989a). The data were derived from an experiment conducted with a dual-beam acoustical system and designed to establish the relationship between the acoustical target strength (TS) of a variety of common zooplanktonic and micronektonic taxa and their length and biomass. These data represent the first extensive set of sound-scattering measurements made on living individuals of these taxa and can help interpret or "calibrate" field sonar-echo data in terms of size frequency distribution of the organisms. Our empirical results are analyzed in the context of recent theoretical studies of zooplankton sound scattering (Stanton 1989a; 1989b) and field studies of zooplankton and micronekton ecology using multiple-frequency and dual-beam acoustical techniques.

\section{METHODS}

The acoustical experiments were conducted at Friday Harbor Laboratories, Friday Harbor (FHL), Washington during the period 8-28 July 1987. A modified BioSonics model 105 echo sounder equipped with a $420-\mathrm{kHz}$ dualbeam transducer was used for all of the experiments (Fig. 1). The dual-beam transducer was of a new coaxial design transmitting with a $3^{\circ}$ narrow-beam ring element and receiving with both that element as well as a $10^{\circ}$ wide-beam central element. The ceramic transducer elements were potted in

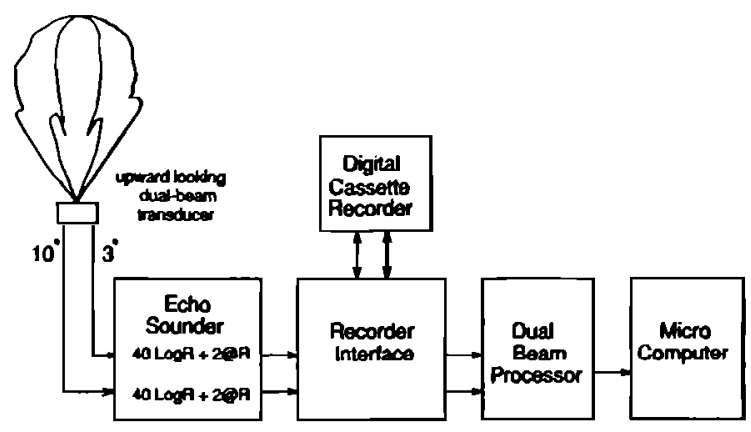

FIG. 1. Schematic diagram of BioSonics, Inc. $420-\mathrm{kHz}$ dual-beam echo sounding system used in the Friday Harbor experiments.
TABLE I. Calibration data for 420-kHz echosounder.

\begin{tabular}{lc}
\hline \hline Transducer nominal beamwidth: & $3^{\circ} / 10^{\circ}$ \\
Directivity index (narrow beam): & $38.09 \mathrm{~dB}$ \\
Composite directivity: & $31.85 \mathrm{~dB}$ \\
Wide beam dropoff: & $1.104 \mathrm{~dB}$ \\
Source level: & $216.4 \mathrm{~dB}$ re: $1 \mu \mathrm{Pa}$ at $1 \mathrm{~m}$ \\
Receiver sensitivity: & $-136.6 \mathrm{~dB}$ re: $1 \mathrm{~V} / \mu \mathrm{Pa}$ \\
\hline
\end{tabular}

epoxy and encased in an anodized aluminum housing. The diameter $D$ of the narrow-beam element was $7.30 \mathrm{~cm}$ giving a near-field distance $\left(D^{2} / \lambda\right)$ of $1.48 \mathrm{~m}$ (where $\lambda$ is the wavelength). For each transmission, the difference in received echo intensity between narrow- and wide-beam elements was used to calculate the polar angular position of the target within the beam. This positional information was then used during signal processing to remove the beam-pattern effects in the determination of TS.

Calibration of the echo sounder and transducer performance was done in the BioSonics calibration tank before and after the series of experiments. Calibration data provided system performance parameters for source level, receiver sensitivity, and transducer beam pattern (Table I). For all experiments, the pulse length was $0.3 \mathrm{~ms}$. With this pulse length, single targets could be detected if their distance from a nearest neighbor, perpendicular to the transducer, was greater than approximately $26 \mathrm{~cm}$.

Acoustical measurements were made on individual animals placed in a cylindrical enclosure deployed off the FHL dock (Fig. 2). The enclosure was $2 \mathrm{~m}$ in diameter by $3 \mathrm{~m}$ in

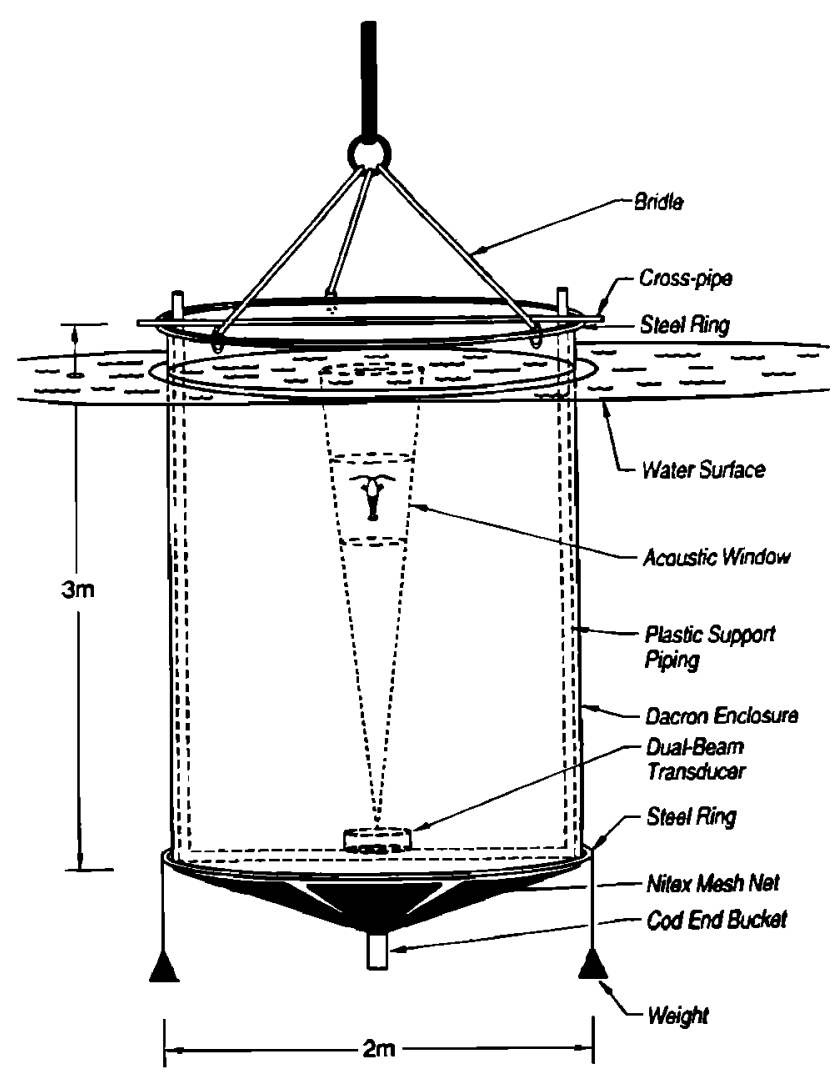

FIG. 2. Schematic drawing of the Friday Harbor Experimental Chamber. The enclosure was deployed off the laboratory dock. See text for details. 
depth and was constructed of black Dacron cloth. Sewn to the bottom of the enclosure was a conical net of $53-\mu \mathrm{m}$ mesh to which a 10-cm-diam cod-end bucket was fixed. Stainless steel rings were attached externally to the top and bottom of the enclosure to provide support for a three-part stainless steel bridle at the top and three $11-\mathrm{kg}$ weights at the bottom. The transducer was positioned facing upward in the center of the enclosure at the bottom about $2.5 \mathrm{~m}$ from the water's surface.

The enclosure was lowered into the seawater $\left(\sim 10^{\circ} \mathrm{C}\right)$ by means of a mechanical hoist and boom located at the end of the FHL dock. Water filled the enclosure through the 53$\mu \mathrm{m}$ mesh providing the filtered seawater in which the experiments were performed. Experiments were conducted at slack tide when water movements around the enclosure were minimal.

A metal pipe was fixed across the center of the upper stainless steel ring and above the water line to provide a point of attachment for tethered acoustical targets or a tethered stainless steel ball used for locating the center of the acoustical beam. The tethered steel ball was used as a reference target, to monitor equipment performance during the experiments, and for locating the enclosure's "acoustical window." The acoustical window, defined as a volume in which the target strengths of individual animals could be measured accurately, was limited to a region between approximately 1.5 and $2.2 \mathrm{~m}$ above the transducer. The lower limit of the acoustical window was set by the transducer's near-field effects; the upper limit was set by a region of elevated noise resulting from side-lobe reflections off the enclosure's walls and the surface.

A variety of planktonic and nearshore crustaceans were collected from San Juan Channel and near the FHL breakwater for the experiments (Table II). In addition, experiments were conducted on several specimens of the jelly fish, Aequorea victoria, and a single individual of the ctenophore, Bolinopsis sp., also collected near the breakwater. Individual crustaceans approximately $10 \mathrm{~mm}$ and larger were tethered with $10-\mu \mathrm{m}$ monofilament nylon line tied around their thorax and suspended in the enclosure's acoustic window. The line, by itself, was not detectable with the dual-beam system. Small copepods, mysids, and an amphipod were inserted into the acoustic window with a $1-\mathrm{m}$-long glass pipette that was removed prior to making measurements. Gelatinous zooplankton were maneuvered into the acoustic window with the pipette. All but one of the individuals used in the experiments were alive, although most were anesthetized in a dilute alcohol-seawater solution prior to experiments.

With an individual situated in the acoustical window, TS measurements were collected for 1 to $3 \mathrm{~min}$. In most of the experiments the ping rate was 30 pings/s. Depending upon the background noise in the enclosure, this usually resulted in 100 to 1000 independent echoes for estimating TS characteristics. For several of the larger crustaceans, two or three experimental runs were made with the animal in different orientations. Typically, during the first run, the animal's orientation was ventral towards the transducer, and during the second run, the orientation was dorsal towards the transducer. For smaller crustaceans, the orientation during a run
TABLE II. Species used in the Friday Harbor experiments. All animals listed are crustaceans except for the Cnidaria and Ctenophora.

\begin{tabular}{|c|c|c|c|}
\hline Taxa group & Species name & $\begin{array}{c}\text { Individual I.D. } \\
\text { \#'s used } \\
\text { in experiments }\end{array}$ & $\begin{array}{c}\text { Size } \\
\text { range }(\mathrm{mm})\end{array}$ \\
\hline Cnidaria & Aequorea victoria & $42,43,45$ & $20-55$ \\
\hline Ctenophora & Bolinopsis sp. & 44 & 45 \\
\hline Amphipoda & $\begin{array}{l}\text { Cyphocaris } \\
\text { challengeri }\end{array}$ & 16 & 10 \\
\hline Copepoda & $\begin{array}{l}\text { Neocalanus } \\
\text { cristatus } \\
\text { Euchaeta } \\
\text { elongata }\end{array}$ & $\begin{array}{l}35(\text { dead }), 36 \\
28\end{array}$ & $\begin{array}{l}8 \\
5\end{array}$ \\
\hline Mysidacea & Neomysis rayii & $\begin{array}{l}8,11,12,13,17 \\
18,19,20,22,23 \\
24,26,31,32,34 \\
37,50\end{array}$ & $13-31$ \\
\hline Euphausia & $\begin{array}{l}\text { Euphausia } \\
\text { pacifica }\end{array}$ & 7,9 & $19-21$ \\
\hline \multirow[t]{2}{*}{ Decapoda } & $\begin{array}{l}\text { Pandalus danae } \\
\text { Crangon }\end{array}$ & $27,38,39,40,46,56$ & $46-90$ \\
\hline & $\begin{array}{l}\text { communis } \\
\text { Hippolyte clarki }\end{array}$ & $\begin{array}{l}33,48 \\
47,49,51,52,53 \\
54,55\end{array}$ & $\begin{array}{l}10-24 \\
17-38\end{array}$ \\
\hline
\end{tabular}

was often quite variable because of their locomotion.

Returning echoes were recorded digitally on a Sony Beta Max $\frac{1}{2}$-in. VCR tape during the course of an experiment. In the laboratory, these data were played back through a BioSonics model 171 tape recorder interface to the dualbeam processor and microcomputer for analysis. Echoes that were derived from within the acoustic window and that fulfilled single target criteria (as opposed to narrower noise peaks and broader multiple echoes) were recorded in a computer file. These echoes were processed to determine backscattering cross section $\left(\sigma_{\mathrm{bs}}\right)$ and target strength (TS), where TS $=10 \log \left(\sigma_{\mathrm{bs}}\right)$ by the above-mentioned procedure. Note that throughout the manuscript, $\sigma_{\text {bs }}$ is computed relative to a unit reference area of $1 \mathrm{~m}^{2}$. Finally for each individual, we obtained an average value of the backscattering cross section $\left(\bar{\sigma}_{\mathrm{bs}}\right)$

$$
\bar{\sigma}_{\mathrm{bs}}=\frac{1}{N} \sum_{i=1}^{N} \sigma_{\mathrm{bs}_{i}}
$$

target strength based on the average $\sigma_{\mathrm{bs}}, \mathrm{TS}_{\bar{\sigma}} \equiv 10 \log \left(\bar{\sigma}_{\mathrm{bs}}\right)$, and the average target strength (i.e., $\overline{10 \log \sigma_{b s}}$ ), where

$$
\overline{\mathrm{TS}} \equiv \overline{10 \log \sigma_{\mathrm{bs}}}=\frac{10}{N} \sum_{i=1}^{N} \log \left(\sigma_{\mathrm{bs}_{i}}\right),
$$

as well as distributions of TS and $\sqrt{\sigma_{\mathrm{bs}}}$ (echo amplitude).

\section{RESULTS}

\section{A. Relationships between TS and length, wet weight, dry weight, $k a$}

Fifty-two sets of target strength data were acquired on 39 different individual crustaceans including amphipods, copepods, decapods, euphausiids, and mysids (Table III). 
TABLE III. The TS frequency distributions for each individual animal and in some cases replicate acoustical characterizations. Species ordered by increasing acoustical size. Replicate sets of echoes from a number of individuals are indicated by the decimal fraction on the I.D. number.

\begin{tabular}{|c|c|c|c|c|c|c|c|c|c|c|c|c|c|c|c|c|c|c|c|c|c|c|c|c|c|c|}
\hline I.D. \# - 95 & -93 & -91 & -89 & -87 & -85 & -83 & -81 & -79 & -77 & -75 & -73 & -71 & -69 & -67 & -65 & -63 & -61 & -59 & -57 & -55 & -53 & -51 & -49 & -47 & -45 & -43 \\
\hline 28 & 3 & 2 & 2 & 1 & 3 & 1 & & & & & & & & & & & & & & & & & & & & \\
\hline 35 & & & & 1 & 7 & 23 & 52 & 37 & 35 & 15 & 2 & & & & & & & & & & & & & & & \\
\hline 26 & & & & 5 & 15 & 16 & 22 & 32 & 47 & 33 & 7 & 5 & 1 & & & & & & & & & & & & & \\
\hline 24 & & & & 1 & 6 & 13 & 21 & 64 & 55 & 35 & 4 & 2 & & & & & - & & & & & & & & & \\
\hline 13 & & & & & 7 & 9 & 13 & 16 & 14 & 9 & 7 & 2 & & & & & & & & & & & & & & \\
\hline 33 & & & & & & 1 & 0 & 4 & 4 & 62 & 88 & 94 & 27 & & & & & & & & & & & & & \\
\hline 36 & & & & 2 & 3 & 8 & 34 & 51 & 106 & 98 & 73 & 27 & 4 & & & & & & & & & & & & & \\
\hline 11 & & & & 1 & 6 & 2 & 14 & 37 & 51 & 45 & 96 & 76 & 29 & 2 & & & & & & & & & & & & \\
\hline 22 & & & & 1 & 0 & 2 & 7 & 14 & 22 & 14 & 7 & 2 & & & & & & & & & & & & & & \\
\hline 16 & & & & & 1 & 1 & 0 & 7 & 12 & 6 & 6 & 3 & 1 & & & & & & & & & & & & & \\
\hline 50 & & & & & & 12 & 31 & 35 & 63 & 65 & 56 & 28 & 6 & 0 & 0 & 0 & 1 & & & & & & & & & \\
\hline 12 & & & & & & 2 & 15 & 23 & 41 & 40 & 28 & 28 & 10 & 4 & 1 & & & & & & & & & & & \\
\hline 9 & & & & 1 & 9 & 11 & 23 & 36 & 36 & 24 & 20 & 5 & 2 & & & & & & & & & & & & & \\
\hline 37 & & & & & & & 1 & 2 & 2 & 8 & 6 & 10 & 7 & & & & & & & & & & & & & \\
\hline 32 & & & & 2 & 9 & 14 & 17 & 17 & 7 & 2 & & & & & & & & & & & & & & & & \\
\hline 51 & & & & & & 7 & 16 & 41 & 74 & 75 & 96 & 59 & 33 & 13 & 1 & & & & & & & & & & & \\
\hline 17 & & & & 2 & 1 & 5 & 12 & 13 & 18 & 16 & 5 & 4 & & & & & & & & & & & & & & \\
\hline 7 & & & & & 1 & 9 & 10 & 24 & 20 & 24 & 11 & 2 & 1 & 2 & & & & & & & & & & & & \\
\hline 8 & & & & 1 & 6 & 15 & 27 & 29 & 40 & 63 & 39 & 22 & 21 & 3 & & & & & & & & & & & & \\
\hline 20 & & & & 5 & 8 & 39 & 37 & 75 & 123 & 150 & 132 & 75 & 6 & & & & & & & & & & & & & \\
\hline 52 & & & & & & & 1 & 1 & 7 & 16 & 43 & 210 & 823 & 609 & 244 & 30 & & & & & & & & & & \\
\hline 18 & & & & 1 & 1 & 7 & 11 & 27 & 28 & 21 & 13 & 10 & 6 & & & & & & & & & & & & & \\
\hline 19 & & & & 1 & 2 & 5 & 12 & 13 & 28 & 34 & 32 & 9 & 5 & 1 & & & & & & & & & & & & \\
\hline 48 & & & & & 4 & 23 & 48 & 46 & 58 & 30 & 13 & 4 & & & & & & & & & & & & & & \\
\hline 31 & & & & 3 & 9 & 24 & 14 & 25 & 25 & 6 & 8 & 2 & & & & & & & & & & & & & & \\
\hline 34 & & & & & & & & & & & 110 & 408 & 317 & 172 & 51 & 4 & & & & & & & & & & \\
\hline 23 & & & & 1 & 4 & 5 & 14 & 12 & 27 & 33 & 18 & 12 & 2 & & & & & & & & & & & & & \\
\hline 47.1 & & & & & & & & & & & & 4 & 7 & 25 & 65 & 102 & 121 & 23 & 1 & & & & & & & \\
\hline 47.2 & & & & & & & & & & 30 & 129 & 231 & 230 & 224 & 73 & 5 & & & & & & & & & & \\
\hline 53.1 & & & & & & & & & & & 2 & 2 & 38 & 202 & 788 & 986 & 150 & & & & & & & & & \\
\hline 53.2 & & & & & & 2 & 5 & 10 & 24 & 55 & 156 & 381 & 331 & 210 & 62 & 4 & & & & & & & & & & \\
\hline 55.1 & & & & & & & & & & 48 & 91 & 121 & 86 & 92 & 33 & & & & & & & & & & & \\
\hline 49.1 & & & & & 2 & 0 & 6 & 4 & 9 & 21 & 49 & 73 & 121 & 125 & 48 & 8 & 2 & & & & & & & & & \\
\hline 49.2 & & & & & & & & & & & & & 3 & 31 & 95 & 190 & 195 & 369 & 428 & 110 & 1 & & & & & \\
\hline 54.1 & & & & & & & & & & & & & 12 & 85 & 245 & 116 & 6 & 1 & & & & & & & & \\
\hline 54.2 & & & & & & & & & & & & & 14 & 96 & 168 & 70 & 14 & 6 & 6 & 2 & & & & & & \\
\hline 54.3 & & & & & & & 9 & 29 & 85 & 87 & 119 & 90 & 43 & 19 & & & & & & & & & & & & \\
\hline 27.1 & & & & & & & & & & & & & & 19 & 60 & 82 & 24 & 16 & 1 & & & & & & & \\
\hline 27.2 & & & & & & & & & & & & & 3 & 35 & 122 & 229 & 268 & 346 & 672 & 259 & & & & & & \\
\hline 40.1 & & & & & & & & & & & & & & 11 & 34 & 63 & 94 & 139 & 216 & 294 & 397 & 277 & 139 & 17 & 2 & 3 \\
\hline 40.2 & & & & & & & & & & & & & 2 & 6 & 19 & 16 & 38 & 63 & 67 & 105 & 216 & 330 & 284 & 265 & 90 & 44 \\
\hline 38.1 & & & & & & & & & & & & & 3 & 11 & 49 & 80 & 127 & 160 & 138 & 113 & 30 & 15 & & & & \\
\hline 38.2 & & & & & & & & & & & & & 8 & 33 & 122 & 224 & 211 & 51 & & & & & & & & \\
\hline 46.1 & & & & & & & & & & & & & 3 & 15 & 31 & 85 & 124 & 184 & 231 & 219 & 142 & 6 & & & & \\
\hline 46.2 & & & & & & & & & & & & & 6 & 49 & 86 & 139 & 234 & 305 & 178 & 52 & $s$ & & & & & \\
\hline 56.1 & & & & & & & & & & & & & 5 & 17 & 45 & 83 & 88 & 119 & 140 & 99 & 4 & & & & & \\
\hline 56.2 & & & & & & & & & & & & & & & 2 & 15 & 66 & 211 & 693 & 110 & & & & & & \\
\hline 41.1 & & & & & & & & & & & & & 12 & so & 167 & 296 & 477 & 516 & 438 & 229 & 42 & & & & & \\
\hline 41.2 & & & & & & & & & & & & & 10 & 78 & 251 & 319 & 348 & 280 & 74 & 22 & & & & & & \\
\hline 39.1 & & & & & & & & & & & & & 2 & 15 & 28 & 38 & 69 & 189 & 169 & 138 & 112 & 47 & 11 & & & \\
\hline 39.2 & & & & & & & & & & & & & 2 & 13 & 67 & 155 & 262 & 328 & 179 & 80 & 18 & & & & & \\
\hline
\end{tabular}

When presented as target strengths, a majority of the backscattering data appeared Gaussian, and therefore, parametric statistics were used to describe dispersion about the mean. The consequences of echo-to-echo variation for individual targets in interpreting the results from acoustical field studies are discussed below.
Average TS measurements were used with the logarithmic transformations of the various measures of individual size (length, wet weight, dry weight, and $k a-$ Table IV) to calculate linear functional regression relationships (Ricker, 1973; Jensen 1986; Table V). All regression relationships yielded highly significant, positive correlations (Fig. 3, Ta- 
TABLE IV. Mean target strength $\left[\overline{\mathrm{TS}} \equiv \overline{10 \log \left(\sigma_{\mathrm{bs}}\right)}\right]$, mean backscattering cross section ( $\left.\overline{\sigma_{\mathrm{bs}}}\right)$, and size measurements for each individual listed in Table II. [Note that $\overline{\mathrm{TS}} \neq 10 \log \left(\overline{\sigma_{\mathrm{bs}}}\right)$; units of all $\sigma_{\mathrm{bs}}$ in $\mathrm{m}^{2}$.]

\begin{tabular}{|c|c|c|c|c|c|}
\hline taxa I.D.\# & $\begin{array}{c}\overline{T S} \\
(\mathrm{~dB})\end{array}$ & $\overline{\sigma_{\mathrm{bs}}}$ & $\begin{array}{l}\text { Length } \\
(\mathrm{mm})\end{array}$ & $\begin{array}{l}\text { Wet weight } \\
\text { (mg) }\end{array}$ & $\begin{array}{l}\text { Dry weight } \\
\text { (mg) }\end{array}$ \\
\hline \multicolumn{6}{|c|}{ Crustacean zooplankton } \\
\hline 7 & -77.08 & $2.754 E-08$ & 21 & 84.0 & 14.0 \\
\hline 8 & -75.84 & $3.972 E-08$ & 21 & 69.0 & 14.0 \\
\hline 9 & -77.87 & $2.259 E-08$ & 19 & 56.0 & 9.0 \\
\hline 11 & -74.33 & $4.932 E-08$ & 14 & 15.0 & 4.0 \\
\hline 12 & -77.05 & $2.723 E-08$ & 18 & 52.0 & 8.0 \\
\hline 13 & -78.76 & $1.858 E-08$ & 14 & 23.0 & 3.5 \\
\hline 16 & -76.07 & $3.097 E-08$ & 10 & 22.0 & 5.0 \\
\hline 17 & -77.76 & $2.249 E-08$ & 18 & 65.0 & 11.5 \\
\hline 18 & -76.68 & $3.076 E-08$ & 23 & 100.0 & 18.7 \\
\hline 19 & -75.83 & $3.532 E-08$ & 27 & 113.0 & 23.1 \\
\hline 20 & -76.00 & $3.304 E-08$ & 22 & 76.0 & 15.0 \\
\hline 22 & -77.22 & $2.333 E-08$ & 13 & 25.2 & 4.5 \\
\hline 23 & -76.40 & $3.083 E-08$ & 30 & 167.2 & 35.9 \\
\hline 24 & -76.29 & $2.825 E-08$ & 13 & 18.7 & 3.2 \\
\hline 26 & -78.50 & $1.963 E-08$ & 12 & 13.9 & 2.4 \\
\hline 27.1 & -63.44 & $5.117 E-07$ & 46 & 1099.0 & 270.0 \\
\hline 27.2 & -59.06 & $1.521 E-06$ & 46 & 1099.0 & 270.0 \\
\hline 28 & -88.52 & $1.803 E-09$ & 5 & 9.0 & 1.5 \\
\hline 31 & -79.49 & $1.611 E-08$ & 31 & 136.3 & 30.2 \\
\hline 32 & -80.94 & $9.817 E-09$ & 19 & 43.6 & 9.5 \\
\hline 33 & -72.58 & $6.138 E-08$ & 10 & 13.6 & 3.5 \\
\hline 34 & -69.63 & $1.213 E-07$ & 29 & 128.3 & 31.3 \\
\hline 35 & -79.54 & $1.334 E-08$ & 8 & 10.2 & 1.9 \\
\hline 36 & -76.11 & $3.076 E-08$ & 8 & 12.4 & 3.7 \\
\hline 37 & -72.86 & $6.501 E-08$ & 20 & 42.9 & 9.2 \\
\hline 38.1 & -58.96 & $1.722 E-06$ & 46 & 770.4 & 452.3 \\
\hline 38.2 & -62.63 & $6.026 E-07$ & 46 & 1770.4 & 452.3 \\
\hline 39.1 & -57.25 & $2.618 E-06$ & 49 & 2497.4 & 631.1 \\
\hline 39.2 & -59.83 & $1.271 E-06$ & 49 & 2497.4 & 631.1 \\
\hline 40.1 & -54.75 & $4.808 E-06$ & 90 & 7942.7 & 2802.5 \\
\hline 40.2 & -51.16 & $1.156 E-05$ & 90 & 7942.7 & 2802.5 \\
\hline 41.1 & -59.72 & $1.355 E-06$ & 57 & 2767.8 & 594.4 \\
\hline 41.2 & -61.86 & $7.889 E-07$ & 57 & 2767.8 & 594.4 \\
\hline 46.1 & -57.73 & $2.188 E-06$ & 50 & 2166.4 & 519.5 \\
\hline 46.2 & -60.36 & $1.140 E-06$ & 50 & 2166.4 & 519.5 \\
\hline 47.1 & -62.90 & $5.821 E-07$ & 31 & 315.5 & 64.6 \\
\hline 47.2 & -69.43 & $1.346 E-07$ & 31 & 315.5 & 64.6 \\
\hline 48 & -78.47 & $1.811 E-08$ & 24 & 145.0 & 25.6 \\
\hline 49.1 & -69.41 & $1.479 E-07$ & 38 & 689.8 & 128.3 \\
\hline 49.2 & -59.50 & $1.358 E-06$ & 38 & 689.8 & 128.3 \\
\hline 50 & -77.97 & $2.213 E-08$ & 17 & 35.7 & 5.7 \\
\hline 51 & -74.35 & $5.035 E-08$ & 17 & 58.0 & 10.9 \\
\hline 52 & -68.18 & $1.683 E-07$ & 21 & 101.2 & 17.4 \\
\hline 53.1 & -64.04 & $4.198 E-07$ & 36 & 523.3 & 90.9 \\
\hline 53.2 & -70.13 & $1.167 E-07$ & 36 & 523.3 & 90.9 \\
\hline 54.1 & -64.92 & $3.396 E-07$ & 36 & 528.5 & 132.7 \\
\hline 54.2 & -64.85 & $3.802 E-07$ & 36 & 528.5 & 132.7 \\
\hline 54.3 & -73.60 & $5.598 E-08$ & 36 & 528.5 & 132.7 \\
\hline 55.1 & -67.99 & $1.745 E-07$ & 37 & 462.2 & 112.7 \\
\hline 55.2 & -70.19 & $1.168 E-07$ & 37 & 462.2 & 112.7 \\
\hline 56.1 & -59.49 & $1.455 E-06$ & 53 & 2076.1 & 553.1 \\
\hline 56.2 & -57.42 & $1.910 E-06$ & 53 & 2076.1 & 553.1 \\
\hline \multicolumn{6}{|c|}{ Gelatinous zooplankton } \\
\hline 42 & -62.77 & $7.998 E-07$ & 50 & 12679.0 & 458.7 \\
\hline 43 & -73.13 & $7.925 E-08$ & 55 & 20220.4 & 729.1 \\
\hline 44 & -80.03 & $1.337 E-08$ & 45 & 17267.3 & 617.3 \\
\hline 45 & -70.56 & $1.164 E-07$ & 20 & 2686.8 & 99.1 \\
\hline
\end{tabular}

ble V). The relationship between TS and dry weight exhibited the strongest correlation, i.e., exhibited the lowest variance, followed by wet weight and $k a_{s}$, and then length. The slopes estimated by these procedures imply that backscatter- ing cross section increases with the volume of the animal rather than its cross-sectional area. The consequences of this relationship in distinguishing between alternative theoretical scattering models will be discussed below. 
TABLE V. Left side: Functional regression equations relating mean target strength ( $\overline{\mathrm{TS}}$ ) of crustacean zooplankton at $420 \mathrm{kHz}$ to their length (mm), wet weight $(\mathrm{mg})$, dry weight $(\mathrm{mg})$, and $k a_{s}$ (wave number $\times$ equivalent spherical radius). Here, $N$ is the number of observations; $R$ the correlation coefficient; $P$ the significance level; $\boldsymbol{P}<0.05$ indicates the slope is significantly different from zero. Right side: Coefficient to $\log \operatorname{corithms}$ [ $\log$ (length), etc.] as derived by acoustic scattering theory for comparison with regression coefficients on left-hand side. Since $k$ was constant in the experiment, the coefficient 30.84 in the fourth line corresponds to variations in $a_{s}$, not $k a_{s}$.

\begin{tabular}{|c|c|c|c|c|c|}
\hline \multirow[b]{2}{*}{ Equation } & \multirow[b]{2}{*}{$N$} & \multirow[b]{2}{*}{$R$} & \multirow[b]{2}{*}{$P$ level } & \multicolumn{2}{|c|}{$\begin{array}{l}\text { Scattering theory } \\
\text { Coefficient to } \log \text { (variable) }\end{array}$} \\
\hline & & & & $\begin{array}{l}\text { Straight finite } \\
\text { cylinder }\end{array}$ & Sphere \\
\hline$\overline{\mathrm{TS}}=-114.18+31.11 \log ($ length $)$ & 52 & 0.85 & $<0.001$ & 30 & 20.0 \\
\hline$\overline{\mathrm{TS}}=-93.74+10.27 \log ($ wet weight $)$ & 52 & 0.90 & $<0.001$ & 10 & 6.7 \\
\hline$\overline{\mathrm{TS}}=-85.88+9.68 \log (\mathrm{dry}$ weight $)$ & 52 & 0.91 & $<0.001$ & 10 & 6.7 \\
\hline$\overline{\mathbf{T S}}=-94.93+30.84 \log \left(k a_{s}\right)$ & 52 & 0.90 & $<0.001$ & 30 & 20.0 \\
\hline
\end{tabular}

The average target strengths for the four gelatinous animals (Table III), do not compare favorably with the crustacean wet weight regression and the derivative $k a$ lines given in Fig. 3. For a given wet weight, the target strength of a gelatinous animal appears to be much lower than for a comparably sized crustacean. Based on these data, the use of the crustacean regressions to predict the wet weight or even the length or dry weight of a gelatinous animal will result in an underestimate of its actual physical size. The reason stems in part from the fact that the length to volume relationship for these organisms is quite different from the crustaceans with the gelatinous zooplankton having more volume for a given length. Furthermore, because of their considerably higher water content, gelantinous zooplankton have much less dry weight per unit wet weight. The fit to the TS/dry weight regression is much better than the TS/wet weight regression suggesting that the volume reverberation characteristics of this very limited number of gelatinous animals are most strongly related to their organic matter content which perhaps dominates the acoustic impedance contrast.

\section{B. Frequency distribution of echos from a single individual; sources of variability}

Although the functional regression relationships in Table $\mathrm{V}$ appear to provide reasonably good predictors of crustacean size given a mean TS, understanding the nature of ping-to-ping variability among echoes from a single individual is particularly important if these equations are to be applied to data from the field. Theoretically, if an animal used in our experiments had been either perfectly spherical or any irregular shape and structure and rigidly fixed in place, then its TS measurements should have been distributed as a delta function (i.e., single valued). In our experiments, however, the animals (all nonspherical), while normally having a principal orientation, moved about in the acoustic window. Hence considerable ping-to-ping variability was observed (Table III, Fig. 4) that is due to the fact that TS depends upon both animal orientation and shape, quantities that certainly changed as the animals moved. Additional variability is introduced if there are larger changes in orientation beyond the principal direction. For a number of the larger indi-
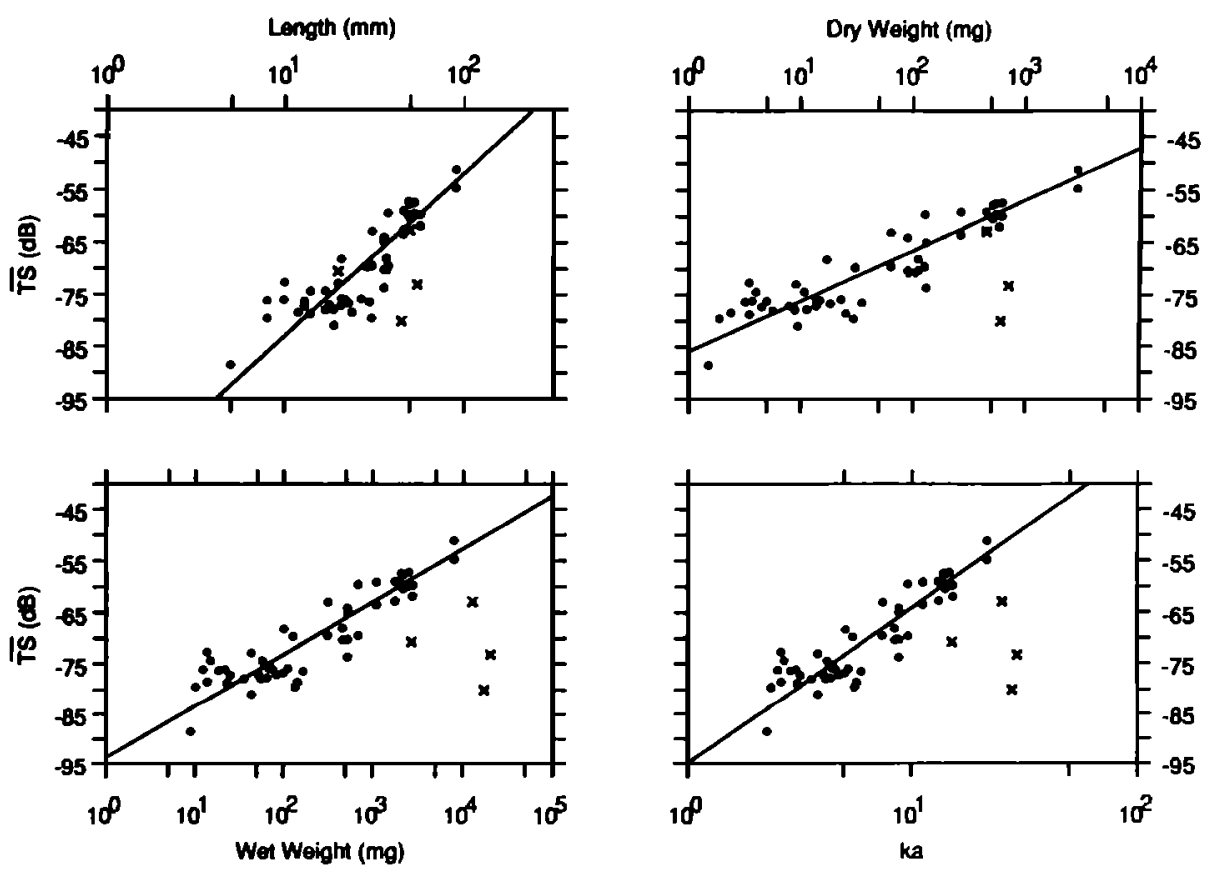

FIG. 3. Plots of mean target strength $\left[\overline{\mathrm{TS}}=\overline{10 \log \left(\sigma_{\mathrm{bs}}\right)}\right]$ for each individual versus the log of their length, wet weight, dry weight, and $k a_{s}$ where $k=2 \pi / \lambda$ and $a_{s}=$ the equivalent spherical radius of the individual (reference area $1 \mathrm{~m}^{2}$ ). Here, $a_{s}$ was computed from the wet weight measurement assuming 1 $\mathrm{g}$ of biomass $=1 \mathrm{cc}$ of seawater. The functional linear regression lines are from the equations given in Table $V$. Note that the points ( $X$ 's) for gelatinous animals were not used in the regression equation computations. Since $k$ was constant in the experiment, the slope of the regression line in the lower righthand plot corresponds to variations in $a_{s}$, not ka. 

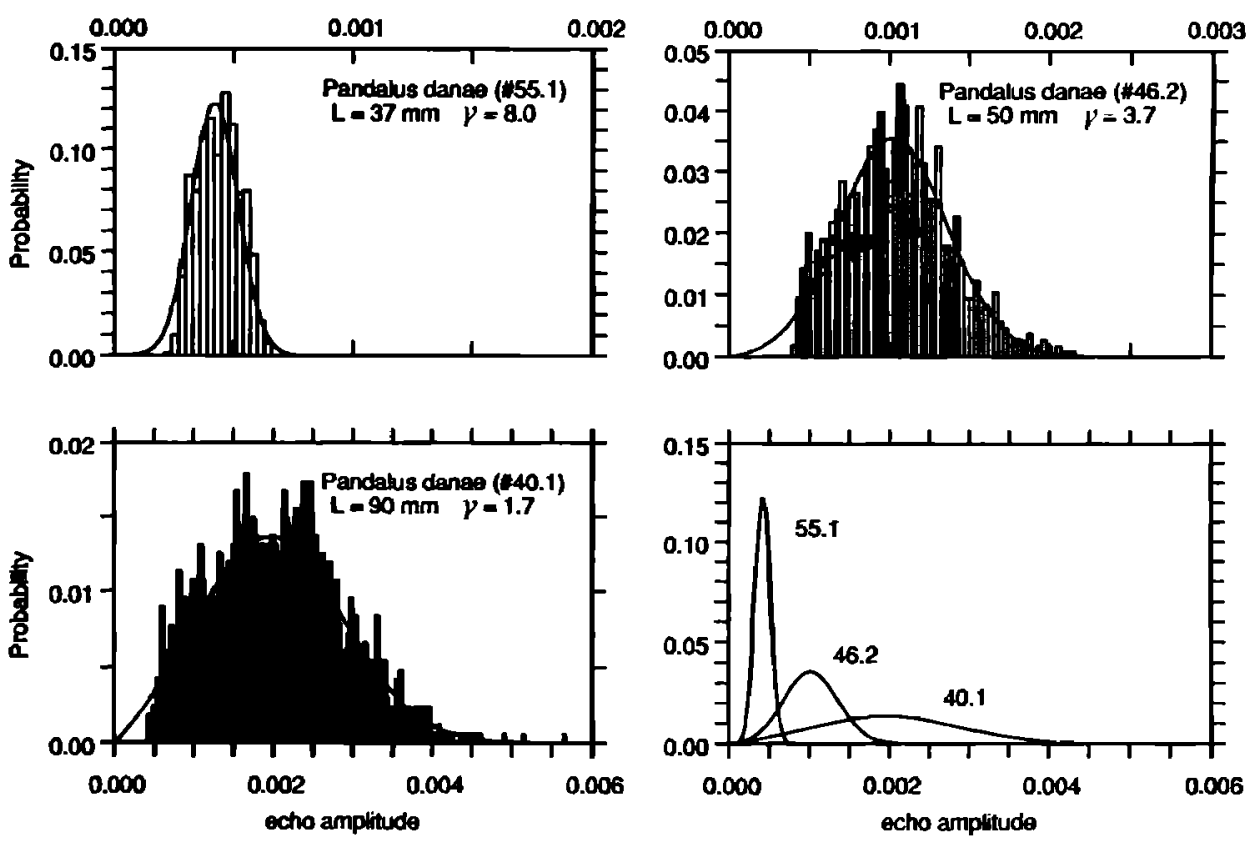

FIG. 4. Probability distributions of echo amplitudes $\left(\sqrt{\sigma_{b s}}\right)$ of three individual decapod shrimp (note $x$-axis scale change). The best-fit Rice PDF is superimposed on each plot. The three Rice PDF curves are also plotted together on a single scale to illustrate the change in $\gamma$ as a function of animal length. viduals, the orientation was reversed, i.e., during the first set of observations the animal was ventral towards the transducer and for a second set, it was dorsal towards the transducer; these frequency distributions are presented separately (Table III).

We have examined this variability by comparing the observed frequency distributions of the echo amplitudes with the Rayleigh, Rice (Rice, 1954), and normal probability density functions (PDF's). In order to perform the statistical analysis on a convenient linear scale, we have used the echo amplitude that is proportional to the square root of the backscattering cross section. The Rice distribution has been suggested as a likely theoretical model (Clay and Heist, 1984; Stanton, in preparation and the Rayleigh and normal PDFs are limiting forms of the Rice PDF.

For each of the 56 data sets ( 52 crustacean plus 4 gelatinous), the observed echo amplitude distributions, their means, and their variances were computed. The Rayleigh (PDF) was computed using the single required parameter, the observed mean backscattering cross section $\bar{\sigma}_{\mathrm{bs}}$ [where $\left.\mathrm{TS} \equiv 10 \log \left(\sigma_{\mathrm{bs}}\right)\right]$ for a given data set. The normal PDF was computed using both the observed mean of the square root of $\sigma_{b s}$ and the associated variance. For the two Rice PDF parameters, $\bar{\sigma}_{\mathrm{bs}}$ from the data (Table IV) was used while " $\gamma$ " (from the notation of Clay and Heist, 1984, which is equal to the ratio of coherently scattered power to incoherently scattered power and describes the shape of the PDF) was determined by iteratively finding the value that minimized the sum of squares of the differences between the observed and theoretical PDF values (Table VI). See the Appendix for additional detail about the Rice PDF. For comparison, this least-squares approach was applied to Rayleigh and normal PDF's as well.

In all cases, the theoretical Rice PDF fit the observed frequency distributions better than did the normal PDF. However, since most distributions were bell shaped, the improvement over the normal in most cases was small. The Rayleigh PDF typically did not fit the observed distributions nearly as well as the other PDFs.

It is not surprising that the Rice PDF provided the best fits since, as mentioned above, it is the most general of the three PDFs. Another advantage of using the Rice PDF as the statistical model for ping-to-ping variation off of a single individual is that, unlike the normal PDF, the distribution is bounded on the lower end by zero, i.e. the echo amplitude will never be negative.

Echo amplitude distributions varied considerably from animal to animal; several examples are illustrated in Fig. 4. Rice PDFs, which were fit to the data by use of the leastsquares method described earlier, are superimposed upon these distributions and also are summarized in a separate panel. The Rice PDFs are shown to fit the data reasonably well indicating not only that the Rice PDF is a good descriptor of the echo fluctuations, but also that the sound scattering by the animals was exhibiting unimodal behavior. The fitted Rice PDFs were found to be narrowest (high $\gamma$ ) for the smallest animal and the widest (low $\gamma$ ) for the largest animal. This trend is due to the fact that, for a given change in animal orientation or shape, variations in phase of the echoes from individual sections of an animal will be greater for the larger animal. Interference effects due to the phase variations of the Huygen "wavelets" cause fluctuations in the amplitude of the "total" echo. Hence, the larger the animal, the greater the degree of fluctuations in the echo. Later in the paper, we will provide a more detailed analysis of the dependence of these fluctuations on the backscattering cross section and then discuss a method for averaging out these fluctuations during field studies. 
TABLE VI. The Rice PDF shape parameter $\gamma$ and the minimized sum (MS) of the squares of the difference between the observed and theoretical values for the Rayleigh, normal, and Rice distributions for each individual listed in Table II. Here, $N$ is the number of observations.

\begin{tabular}{|c|c|c|c|c|c|}
\hline taxa I.D. \# & $\gamma$ & $\begin{array}{c}\text { Rayleigh } \\
\text { MS }\end{array}$ & $\begin{array}{l}\text { Normal } \\
\text { MS }\end{array}$ & $\begin{array}{l}\text { Rice } \\
\text { MS }\end{array}$ & $N$ \\
\hline \multicolumn{6}{|c|}{ Crustacean zooplankton } \\
\hline 7 & 2.9 & 0.02910 & 0.01720 & 0.00886 & 104 \\
\hline 8 & 1.9 & 0.01791 & 0.01421 & 0.01227 & 266 \\
\hline 9 & 2.7 & 0.02334 & 0.01228 & 0.00337 & 167 \\
\hline 11 & 2.4 & 0.01719 & 0.00982 & 0.00508 & 359 \\
\hline 12 & 2.7 & 0.02725 & 0.01527 & 0.00942 & 192 \\
\hline 13 & 2.5 & 0.02905 & 0.02035 & 0.01031 & 77 \\
\hline 16 & 6.6 & 0.06617 & 0.03200 & 0.01683 & 37 \\
\hline 17 & 2.7 & 0.02999 & 0.01866 & 0.00934 & 76 \\
\hline 18 & 2.7 & 0.02841 & 0.01835 & 0.01445 & 125 \\
\hline 19 & 3.6 & 0.02555 & 0.00992 & 0.00284 & 142 \\
\hline 20 & 2.6 & 0.01994 & 0.01041 & 0.00334 & 650 \\
\hline 22 & 6.3 & 0.06598 & 0.03376 & 0.00845 & 69 \\
\hline 23 & 3.0 & 0.02395 & 0.01203 & 0.00521 & 128 \\
\hline 24 & 6.0 & 0.06038 & 0.02918 & 0.00729 & 201 \\
\hline 26 & 2.1 & 0.03215 & 0.02959 & 0.01676 & 179 \\
\hline 27.1 & 9.4 & 0.02701 & 0.00742 & 0.00724 & 202 \\
\hline 27.2 & 2.9 & 0.00681 & 0.00437 & 0.00367 & 1934 \\
\hline 28 & 1.2 & 0.19405 & 0.51337 & 0.17507 & 12 \\
\hline 31 & 3.0 & 0.03354 & 0.02397 & 0.00688 & 116 \\
\hline 32 & 5.4 & 0.09536 & 0.08875 & 0.01352 & 68 \\
\hline 33 & 8.0 & 0.06265 & 0.01799 & 0.00690 & 280 \\
\hline 34 & 9.3 & 0.05568 & 0.01145 & 0.01098 & 1062 \\
\hline 35 & 6.0 & 0.09114 & 0.06807 & 0.01176 & 172 \\
\hline 36 & 4.3 & 0.03766 & 0.01423 & 0.00379 & 406 \\
\hline 37 & 2.7 & 0.02892 & 0.02040 & 0.01458 & 36 \\
\hline 38.1 & 2.0 & 0.00336 & 0.00231 & 0.00222 & 726 \\
\hline 38.2 & 8.1 & 0.02063 & 0.00303 & 0.00247 & 649 \\
\hline 39.1 & 1.7 & 0.00296 & 0.00249 & 0.00235 & 818 \\
\hline 39.2 & 4.3 & 0.00653 & 0.00137 & 0.00142 & 1104 \\
\hline 40.1 & 1.7 & 0.00106 & 0.00067 & 0.00062 & 1676 \\
\hline 40.2 & 1.6 & 0.00078 & 0.00061 & 0.00058 & 1545 \\
\hline 41.1 & 2.8 & 0.00354 & 0.00100 & 0.00094 & 2227 \\
\hline 41.2 & 3.9 & 0.00830 & 0.00203 & 0.00209 & 1382 \\
\hline 46.1 & 2.3 & 0.00265 & 0.00139 & 0.00115 & 1040 \\
\hline 46.2 & 3.7 & 0.00557 & 0.00115 & 0.00114 & 1054 \\
\hline 47.1 & 8.5 & 0.01909 & 0.00201 & 0.00161 & 344 \\
\hline 47.2 & 4.5 & 0.02327 & 0.00455 & 0.00386 & 922 \\
\hline 48 & 3.7 & 0.05191 & 0.02955 & 0.01170 & 226 \\
\hline 49.1 & 4.1 & 0.01504 & 0.00297 & 0.00145 & 468 \\
\hline 49.2 & 3.5 & 0.00633 & 0.00258 & 0.00218 & 1422 \\
\hline 50 & 3.2 & 0.03323 & 0.02166 & 0.00425 & 297 \\
\hline 51 & 2.5 & 0.01675 & 0.00732 & 0.00609 & 415 \\
\hline 52 & 12.0 & 0.04777 & 0.00668 & 0.00382 & 1984 \\
\hline 53.1 & 14.5 & 0.04045 & 0.00578 & 0.00340 & 2168 \\
\hline 53.2 & 5.5 & 0.02678 & 0.00503 & 0.00355 & 1240 \\
\hline 54.1 & 18.6 & 0.05297 & 0.00564 & 0.00249 & 465 \\
\hline 54.2 & 11.0 & 0.03750 & 0.01404 & 0.00911 & 188 \\
\hline 54.3 & 3.4 & 0.02426 & 0.00776 & 0.00611 & 481 \\
\hline 55.1 & 8.0 & 0.03826 & 0.00821 & 0.00407 & 1034 \\
\hline 55.2 & 3.9 & 0.04923 & 0.02320 & 0.01554 & 471 \\
\hline 56.1 & 2.0 & 0.00423 & 0.00325 & 0.00270 & 600 \\
\hline 56.2 & 21.0 & 0.02994 & 0.00922 & 0.00810 & 1097 \\
\hline \multicolumn{6}{|c|}{ Gelatinous zooplankton } \\
\hline 42 & 0.7 & 0.00419 & 0.00473 & 0.00416 & 574 \\
\hline 43 & 1.2 & 0.01068 & 0.0113 & 0.00944 & 150 \\
\hline 44 & 2.9 & 0.09365 & 0.07936 & 0.06572 & 32 \\
\hline 45 & 2.5 & 0.01431 & 0.00787 & 0.00628 & 140 \\
\hline
\end{tabular}

\section{DISCUSSION AND FURTHER ANALYSIS}

\section{A. TS relationships to length and weight measures}

Our empirical results with live zooplankton and micronekton are consistent with the findings of previous inves- tigators (Greenlaw, 1977; Richter, 1985a). The slopes of our regression relationships are virtually identical to those of Richter (1985a), despite the fact that his regressions were based on preserved animals. These results confirm Richter's (1985a) conclusion that backscattering cross section of zooplankton increases with volume rather than cross-sectional area.

The intercepts of the regression relationships are significantly higher by approximately $5 \mathrm{~dB}$ than Richter's (1985a) indicating that preserved animals are weaker acoustical targets than live animals. Richter (1985a) suggested such a relationship based on his limited measurements of live euphausiids. Therefore, we also can confirm his suggestion that preservation reduces an animal's target strength.

In addition to their value in comparisons with previous data, our empirical results have important implications for testing theoretical scattering models and interpreting the results from acoustical field studies. The remainder of this discussion will be devoted to those two topics.

\section{B. Theoretical implications: Cylinder scattering models}

\section{Backscattering cross sections}

Until very recently, most researchers in the field of zooplankton and micronekton bioacoustics have assumed that a fluid sphere model (Anderson, 1950; Johnson, 1977) adequately characterizes acoustic scattering from these animals. Our data as well as those previously published for zooplankton and micronekton (Greenlaw, 1977; Richter, 1985a) are in conflict with the predictions of fluid sphere models (Stanton, 1988, 1989a, 1989b). On a plot of reduced TS vs $k a$, these spherical models predict modal oscillations and a leveling off of the trend of reduced TS at $k a>1$ (beyond the transition from the Rayleigh to the geometric scattering region-Fig. 5). The trend of leveling off would indicate that at $k a>1$, the backscattering cross section increases with the cross-sectional area of the animal rather than its volume. Richter's (1985a) results and our results led each of us to conclude just the opposite. Qualitatively, the general trend of the scattering increases with $k a$ rather than leveling off which indicates that the animals are not scattering sound like spheres. Quantitatively, the regression relationships given in Table $V$ demonstrate that the backscattering cross section is approximately proportional to the volume rather than the cross-sectional area. Without making any assumptions, for the cross section to be exactly proportional to volume, the slopes in wet weight (which, for these nearly neutrally buoyant animals, is proportional to volume) and $k a_{s}$ regressions (rows 2 and 4 of the table, left side) would have equaled 10 and 30 , respectively. Had the scattering depended upon area, then the slopes would have equaled 6.7 and 20 , respectively. The empirical fits to the 52 data points are 10.27 and 30.84 which, to within the spread of the data, indicate that the scattering essentially depends upon the volume.

By making certain assumptions, we can analyze Table $\mathrm{V}$ even further (i.e., rows 1 and 3 ). Row 1 of Table $V$ : If one can assume that animal length is proportional to equivalent spherical radius (i.e., that the lengthwise and cross-sectional dimensions of the animals maintain the same relative pro- 
portions, regardless of length) then for the backscattering cross section to be proportional to volume, the slope of the TS by length regression in row 1 (left side) of Table V should be 30. If the scattering depends upon cross-sectional area, then the slope should be 20 . The empirically derived slope of 31.11 again suggests a dependence upon volume. It is also interesting to note that while the slopes in the length and $k a$ (rows 1 and 4) are each slightly higher than 30 , they are very close to each other, which indirectly verifies the assumption of the length being proportional to equivalent spherical radius. Row 3: Since crustacean dry weight is proportional to wet weight (which is proportional to volume), ideally, for the scattering to depend upon volume, the slope of the TS by dry weight regression of row 3 should be 10 . As in the wet weight case, for the scattering to depend upon cross-sectional area, the slope should be 6.7. The regression analysis yielded a slope of 9.68 that is indistinguishable, given the spread of the data, from the volume-dependent scattering prediction.

Because of the overwhelming evidence that the scattering depends upon the volume of a crustacean rather than its cross-sectional area, we must look for more accurate models than the spherical scattering models used to date. In a series of recent papers, Stanton (1988; 1989a; 1989b) found that scattering by decapod shrimp and euphausiids, which are both elongated, could be much better described by a model that assumes a cylindrical shape rather than a spherical one. The relationship between reduced TS and $k a$ predicted by cylinder models matches the shrimp and euphausiid scattering data published by Greenlaw (1977) far better than the relationship predicted by sphere models. The shrimp were best modeled as straight finite length cylinders whose length corresponded approximately to thorax length (Stanton, 1988). The euphausiids were best modeled as uniformly bent finite-length cylinders whose length corresponded approximately to total length (Stanton, 1989a). Because of the mathematical complexity of the models, Stanton (1989b) reduced them to simple approximate forms. In addition to the good fit between Greenlaw's data and the cylinder models, the latter also predicted an orientation dependence of the scattering which is what one would expect for the elongate organisms.

In order to investigate the volumetric dependence of the scattering, we use the high-frequency ( $k a \gg 1)$ limits of the sphere and straight cylinder models as given in approximate form by Stanton (1989b):

$$
\begin{array}{ll}
\sigma_{\mathrm{bs}} \approx a_{s}^{2} R^{2} / 4 \quad & \text { (sphere, } \left.k a_{s} \gg 1\right), \\
& \text { (straight cylinder, } \\
\sigma_{\mathrm{bs}} \approx L^{2} k a_{c} R^{2} / 4 \pi & \text { broadside incidence } \\
& \left.k a_{c} \gg 1\right),
\end{array}
$$

where $a_{s}$ is the spherical radius, $a_{c}$ the cylindrical radius, $L$ the length of the cylinder, and $R$ the Rayleigh plane-wave/ plane-interface reflection coefficient. Both equations represent the trend of the backscattering and are essentially "smoothed" versions of the exact (oscillatory) modal series solutions. If one assumes that the length of the organism is proportional to its (equivalent) cylindrical radius (i.e., the relative proportions of the dimensions of the organisms re- main the same regardless of size), then Eq. (1b) can be rewritten as follows:

$$
\sigma_{\mathrm{bs}}=\left(B k R^{2} / 4 \pi^{2}\right) V,
$$

where $B$ is the proportionality constant between $L$ and $a_{c}$ ( $L=B a_{c}$ ) and $V\left(=\pi a_{c}^{2} L\right)$ is the volume of the organism. Converting Eq. (2) to TS:

$$
\begin{aligned}
& \text { TS } \equiv 10 \log \left(\sigma_{\mathrm{bs}}\right), \\
& \mathrm{TS}=10 \log \left(B k R^{2} / 4 \pi^{2}\right)+10 \log (V),
\end{aligned}
$$

where the direct dependence of the volume is derived. Note that in Eq. (3) and later equations, the arguments of the logarithm within each equation collectively have the dimensions of $\mathrm{m}^{2}$. By relating the volume to wet weight and dry weight by a proportionality constants, $E$ and $D$, respectively, the TS can be expressed in terms of these weights as follows:

$$
\begin{aligned}
& \mathrm{TS} \approx 10 \log \left(B E k R^{2} / 4 \pi^{2}\right)+10 \log \text { (wet weight), (4a) } \\
& \mathrm{TS} \approx 10 \log \left(B D k R^{2} / 4 \pi^{2}\right)+10 \log \text { (dry weight), (4b) }
\end{aligned}
$$

where the assumed relations $V=E$ (wet weight) and $V=D$ (dry weight) were used. The coefficient 10 of the second logarithm in each equation should be compared with the empirically derived slopes, 10.27 and 9.68 , in the wet and dry weight regressions given on the left side of rows 2 and 3 of Table V, respectively. In a similar fashion, we can replace $a_{c}$ in Eq. ( 1 b) by $L / B$ to obtain the following relationship:

$$
\sigma_{\mathrm{bs}}=k R^{2} L^{3} / 4 \pi B \text {, }
$$

or in terms of TS:

$$
\text { TS }=10 \log \left(k R^{2} / 4 \pi B\right)+30 \log (L),
$$

where the coefficient 30 , which is multiplied by the logarithm of length in this equation, should be compared with the empirically derived slope, 31.11 , in the TS by length regression given in Table $\mathbf{V}$.

Finally, from Eq. (2), we now relate the cylinder scattering model to the equivalent spherical radius using $V=(4 / 3) \pi a_{s}^{3}$ :

$$
\sigma_{\mathrm{bs}}=B R^{2}\left(k a_{s}\right)^{3} / 3 \pi k^{2},
$$

or in terms of TS as follows:

$$
\mathrm{TS}=10 \log \left(B R^{2} / 3 \pi k^{2}\right)+30 \log \left(k a_{s}\right),
$$

where the coefficient 30 , which is multiplied by the logarithm of $k a_{s}$, should be compared with the empirically derived slope, 30.84, in the TS by $k a_{s}$ regressions given in Table V. (Note that the above equation involving an equivalent spherical radius was based on a cylinder scattering model and should not be confused with the corresponding expression in the next paragraph based on a sphere model.)

The above analysis, which was based on the cylinder scattering model given in Eq. (1b), is summarized in the right side of Table $V$. When repeating the analysis using the sphere model [Eq. (1a)], the target strength can be shown to vary as $20 \log \left(k a_{s}\right), 6.7 \log (V), 20 \log (\mathrm{LENGTH})$, $6.7 \log$ (WET WEIGHT), and $6.7 \log$ (DRY WEIGHT). None of these relationships was observed in the regressions given in Table V.

Because the slopes of the empirically derived regression relationships given in Table $\mathrm{V}$ are so convincingly close to 

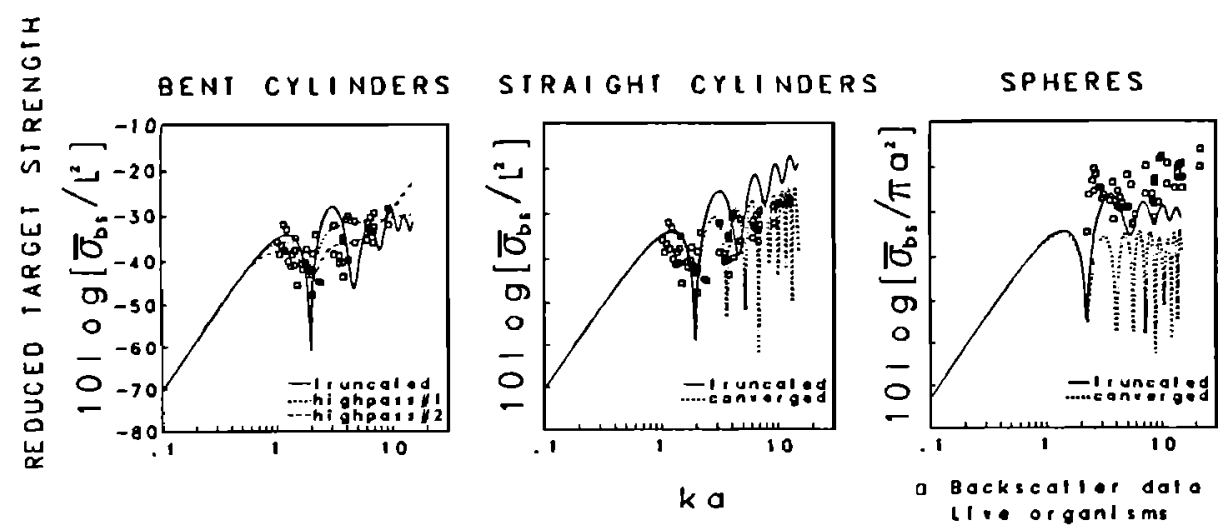

FIG. 5. Plot of the reduced target strength versus $k a$ using the Friday Harbor crustacean data. The data are compared with scattering models involving several shapes where the reduced target strength based on $\bar{\sigma}_{\mathrm{bs}}$ is equal to $10 \log \left(\overline{\sigma_{b s}} / L^{2}\right)$ for the uniformly bent and straight cylinders and $10 \log \left(\overline{\sigma_{b s}} / \pi a^{2}\right)$ for the sphere (where this $a$ is the radius of the sphere, while in $k a, a$ is the cylindrical radius for the cylinders and spherical radius for the spheres. To normalize the data, $a$ was the average equivalent radius calculated from the equations $V=\pi a^{2} L$ and $V=(4 / 3) \pi a^{3}$ for the cylinders and spheres, respectively. Thus for consistency, the data and models involving the cylinders are on different scales than those involving the spheres (note the elevated level of the data with the sphere normalization). Modal series solutions involving all shapes are plotted where both converged and truncated (keeping only first two terms of the series) solutions are illustrated and high-pass models involving the bent eylinder with two sets of parameters [ \# 1 to match the peaks of the modal series solution given and \#2 based on parameters determined from laboratory measurements with preserved euphausiids (Stanton, 1989a) ]. As discussed in Stanton (1989), the converged modal solutions correspond to simple shaped (smooth) ideal objects while the truncated solutions can be used in some cases to approximate irregularly shaped or rough objects. See the Appendix for the high-pass model formula and parameter values.

the above slopes predicted by a simple straight cylinder model and in such disagreement with the sphere model, we can conclude that crustaceans are scattering sound much more like cylinders than spheres.

The linear regression analysis should be considered a "first approximation" to an otherwise very complex problem. As shown in previous papers (Anderson, 1950; Stanton $1988,1989 \mathrm{a}, 1989 \mathrm{~b}$ ), the behavior of the backscattering cross section versus $k a$ curve is oscillatory in the $k a>1$ region. Thus the linear regression analysis yields a smoothed version of the actual relationship. To look at this problem in more detail, we now investigate the actual scattering models themselves.

We begin with a comparison between the data and scattering models involving several shapes, uniformly bent and straight finite length cylinders and spheres (Fig. 5). In our comparisons, reduced TS based on $\bar{\sigma}_{b s}$ is plotted against $k a$ where the "reduction" and value of $a$ used depends on whether the shape is cylindrical or spherical (see caption), hence the data in the sphere model plot involve higher values of reduced target strength than those in the cylinder model plots. Most curves represent modal series solutions where each series is either mathematically converged describing a simple smooth shape or prematurely truncated before convergence. The latter has been shown to sometimes approximate the scattering by irregularly shaped objects (Stanton, 1989b). Two "highpass" models for the bent cylinder are also given. They are essentially "smoothed" versions of the oscillatory modal series solutions. Model \#1 is based on matching the peaks of the modal series solution except for near the $k a=2$ null where the width of the data was matched while model \#2 is based mostly on parameters derived from laboratory acoustical measurements of preserved euphausiids [Greenlaw (1977) data analyzed in Stanton, 1989b]. See the Appendix for the high-pass model formula and parameter values. Calculations of all curves use density and speed of sound contrasts of live euphausiids as measured by Foote et al. (1990).

The major sources of error in generating these models were the selection of material properties (all shapes) and the determination of bent cylinder shape. It is important to emphasize that the speed of sound contrast possibly depends upon the temperature of the surrounding water; significant errors could have been introduced if the water temperature during our experiments was greatly different than that in Foote et al.'s experiment [they did not publish the temperatures in which their experiments were conducted] (Greenlaw, 1977). The shape was estimated from both what is considered a reasonable value for swimming animals as well as what produced a good fit. Thus the bent cylinder shape was, for the most part, empirically determined from the data.

The differences between the various models that are most important in this particular analysis involve the trend and "variance" in the reduced TS by $k a$ relationship (where the variance represents the variation in data values within a narrow range of $k a$ ). In the empirical results, reduced TS increases with $\mathrm{ka}$ while the variance in this relationship decreases with $k a$. The only model that matches these attributes of the data is the truncated bent cylinder model, the same model that was also found to describe the scattering by preserved euphausiids in Stanton (1989a). At first glance, the data also appear to fit the ideal perfectly smooth (converged) straight cylinder model. However, closer inspection reveals that the variance of the data is not at all consistent 
with that of the model. Finally, neither the trend or general levels of the data coincide well with the sphere models.

There is also an indication of modal interference structure in the data, where the data apparently reproduce the major null predicted by the models at $k a=2$. Beyond that null, it is difficult to distinguish any strong structure in the data, although there seemed to be a downward trend in the data near $k a=5$ and an upward trend near the $k a=6.5$ peak that are consistent with the structure of the bent cylinder model (modal series solution).

Finally, there are two points to emphasize. First, although the overall characteristics of the data are best described by the truncated bent cylinder model in the left-most plot in Fig. 5, the overall trend of the data is nearly the same as the trend of the converged straight cylinder model. This explains why the simple mathematical analysis presented earlier in this section compared so well with the regressions to the data summarized in Table $V$. The variance and overall characteristics of the converged straight cylinder solution are substantially different than those of the data, hence the truncated bent cylinder model gives a better overall fit to the data. Second, the data set is comprised of the means of many backscattering cross-section realizations as each animal changes shape and orientation while swimming. In contrast, the model solutions correspond to single realizations for each $k a$ value with shape and angle of incidence held constant. Thus an improvement over this analysis might be to average ensembles of targets spanning a randomized range of shapes and orientations. The results of such averages would likely affect the trend and modal structure of our data set (data concerning the ranges of shapes and orientations were not collected in this experiment, precluding any related analysis).

In conclusion, regardless of the cylinder model providing the best fit to our data, it is clear that the observed trend of reduced TS increasing with $k a$ is not described by simple sphere models. Hence, the elongation of the animals must be taken into account in developing appropriate scattering models. Also, as demonstrated in the next section, the elongation of the animals strongly influences the ping-toping variability of the echoes.

\section{Echo statistics}

Because the echo amplitude depends upon the shape and orientation of the animals, repeated pings from the same animal will vary in amplitude from ping-to-ping as the animal swims (even after removing beam pattern effects). These variations can cause substantial error in any analysis if they are not taken into account. Hence we now investigate the statistical fluctuations of the echo amplitude.

In a recent theoretical study, a formulation describing the statistical fluctuations of the echo amplitude from randomly rough cylinders was developed (Stanton, submitted and in preparation). In short, the theory, which originally described the scattering by smooth cylinders (Straight, bent, and spheroidal shaped), was extended to include surface irregularities, or texture of the targets. It is believed that such an extension will provide a more realistic basis for describing the scattering of sound by targets that naturally occur in the ocean. The echo fluctuations were described by the Rice PDF and explicit, closed-form expressions involving the straight finite length cylinder were derived to help facilitate description of the fluctuations. In these explicit expressions, the direction of the incident wave was assumed to be normal to the target, and the theory was based on ensembles of realizations of statistically independent stochastically rough cylinders. This theoretical approach is in contrast to the experiments described in this paper where the echo fluctuations were measured from each animal as it changed both shape and orientation. While the explicit form of the theory does not perfectly describe the experiment (note that the more general form does), its simple form is extremely useful in at least qualitatively describing the fluctuations where both the changes in shape and behavior are both combined into an effective stochastic roughness. [Note also that one, in principle, could predict echo fluctuations from a rough sphere model. However, the rough cylinder model is more desirable as it is consistent with the cylinder-like trends of the data described in the previous section as well as having the capability of being used in more general form to directly describe fluctuations induced by changes in orientation (versus indirectly by use of an effective roughness) ].

The low roughness limit of the PDF shape parameter, $\gamma$, was derived in Stanton (in preparation) as follows:

$$
\gamma \approx 3(L / \mathscr{L}) / 32(k \sigma)^{4} \quad(k \sigma \ll 1),
$$

where $\sigma$ is the rms roughness (not to be confused with backscattering cross section) and $\mathscr{L}$ is the correlation length of the roughness as measured along the lengthwise axis of the straight finite cylinder. Assuming that $\mathscr{L}, L$, and $a_{c}$ are all proportional to each other, we derive the approximate formula in terms of the backscattering cross section, $\sigma_{\mathrm{bs}}$ from Eq. (1b):

$$
\gamma \approx A / \sigma_{\mathrm{bs}}^{1.3},
$$

where $\gamma$ is shown to vary inversely with the backscattering cross section raised to a power of 1.3.

Since this equation was derived for a straight cylinder, it may not accurately describe the fluctuations in echo amplitude from deformed organisms. In order to account for the shape of the organisms, we note in the paper by Stanton (1989a) that when the shape is changed, the relationship between $\sigma_{b s}$ and the dimensions of the object may also change, hence changing the power of $\sigma_{\mathrm{bs}}$ in Eq. (10). Rather than deriving an equation describing a shape resembling that of an organism whose boundary conditions we are not yet certain of, we heuristically write a more general formula for $\gamma:$

$$
\gamma \approx A / \sigma_{\mathrm{bs}}^{\mu}, \mu>0,
$$

where for now $\mu$ must be determined empirically from simulations or data.

Equation (11), although general and approximate, shows that for large animals (large cross section), $\gamma$ is small indicating the fluctuations due to changes in shape/orientation are large. Conversely, for small animals (small cross * section), $\gamma$ is large indicating small fluctuations. This finding is intuitively obvious since the larger the objects become with respect to wavelength, the greater the effects of their 


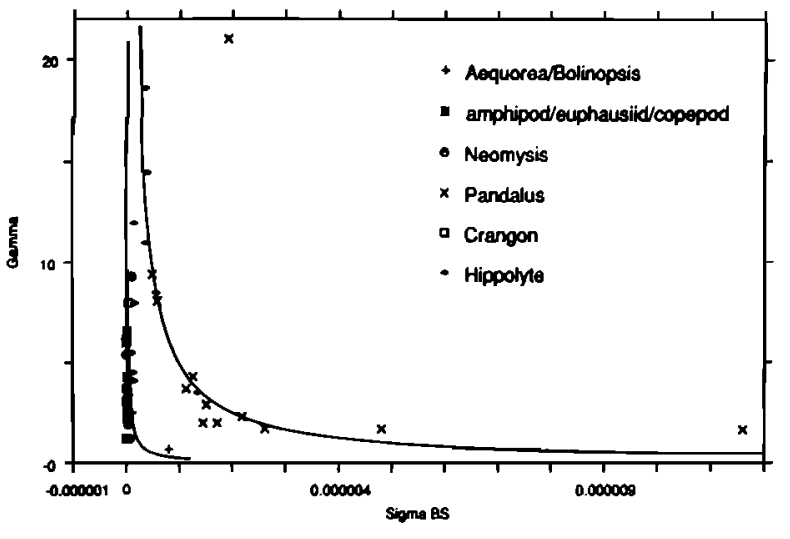

FIG. 6. The relationship between the Rice PDF shape parameter, $\gamma$, and the mean backscattering cross section, $\bar{\sigma}_{\mathrm{bs}}$ for each individual. Also plotted are curves relating $\gamma$ and $\bar{\sigma}_{b s}$ based on Stanton's rough cylinder model work, but with a modified exponent of $\sigma_{\mathrm{bs}}$ that was determined empirically from these data, i.e., $\gamma=A / \sigma_{\mathrm{bs}}$. For the two curves, $A=5.0 \times 10^{-6}$ fits most of the Pandalus and many of the Hippolyte and $A=2.5 \times 10^{-7}$ fits most of the individuals from other taxa.

behavior on the acoustic echo due to various interference phenomena.

In our comparisons of Eq. (11) with the data (Fig. 6), each data point represents the value of $\gamma$ determined from a least-squares fit of the Rice PDF to the distribution of echo amplitudes observed from each animal. Several examples of the best fits to the Rice PDF are given in Fig. 4. Our plots of the fitted $\gamma$ values versus $\sigma_{\mathrm{bs}}$ in Fig. 6 show two subsets of the values to follow the inverse power law as predicted by Eq. (11), with each subset corresponding to a different curve. The parameter $\mu$ was determined empirically to be approximately 1.0 for both curves while $A$ was varied to produce the different curves. We hypothesize that the animals in one subset have a behavioral pattern distinct from those of the other subset (note that while there appears to be good correlation between both subsets of data and each curve on this scale, several curves might describe the smaller group of animals on an expanded scale). Our (in-air) visual observations of the animals during the (underwater) experiments cannot be used to verify or refute this correlation, but the observations were limited for practical reasons-it was very difficult to position an animal in the acoustical window of our experimental enclosure, much less to observe its exact behavior. Furthermore, even had there been high-quality visual observations of behavior, it might have been difficult to distinguish different modes of behavior as the resolution of such observations would have had to be much better than the 0.9$\mathrm{mm}$ quarter wavelength of the sound waves (movements comparable in size to quarter wavelengths cause substantial fluctuations). Either way, the trends of the subsets of data are similar to the trend predicted by the rough cylinder theory indicating the overall stochastic behavior of the scattering and the need to take it into account.

\section{IMPLICATIONS FOR FIELD STUDIES}

Reliable results in field studies using the multiple-frequency inversion technique require an accurate model of in- dividual scattering as a function of $k a$. To date, investigators using this technique have based their scattering models on the assumption that zooplankton and micronekton scatter sound like fluid spheres. As discussed in the previous section, this basic assumption is now being questioned (Stanton, 1988, 1989a, 1989b, this paper). In future field studies, investigators using the multiple-frequency inversion technique will have to determine how robust the results of the method are with respect to deviations from underlying assumptions of the scattering model.

Reliable results in field studies using the dual-beam technique also require an accurate model for converting TS measurements to more conventional measures of animal size. The linear regression relationships presented in Table $\mathrm{V}$ provide a first step towards fulfilling this requirement. In using these relationships, ambiguities arise in the conversion of TS data to conventional size data due to ping-to-ping variability and the modal structure of scattering as a function of size. Such ambiguities result in excessive spread in the size distributions estimated by the dual-beam technique. It is therefore necessary to understand the sources of this variability both empirically and theoretically. In field studies, use of target tracking procedures to get replicate estimates of target strength from single animals will be an important means of increasing the precision of TS measurements and reducing this source of variability.

One of the greatest challenges in using acoustics as a remote sensing tool is to remove as many ambiguities as possible. As demonstrated earlier, because of the fluctuations in echo amplitude that arise from the changes in animal shape and orientation, a small organism may produce a stronger echo than a larger organism in a given realization (Figs. 4 and 6). In addition, even after the backscatter data are averaged, the modal interference in the backscattering can cause the mean backscattering cross section from a smaller animal to be larger than that from a larger animal (Fig. 5). By assuming a scattering model that contains the modal interference structure and includes the mean over an ensemble of realizations of animal shapes and orientations, one can invert multiple-frequency sonar data to estimate the size distribution of the animal assemblage. When one uses a singlefrequency system such as the one described in this article, however, one does not have comparable information to indicate where the animal is on the backscattering versus $\mathrm{ka}$ curve. Hence the enhanced potential for ambiguities when one uses a single-frequency system. It is conceivable that as few as two frequencies operating in a dual-beam mode could resolve such ambiguities.

Due to the simpler design and operating considerations associated with single-frequency systems, it is important to design a sampling scheme that avoids as many ambiguities as possible. As the modal interference structure and echo statistics pose the greatest problem, those areas must be addressed. When accurate estimates of animal size are important, it is essential to operate the acoustical system in a region where there is the least modal structure and most consistent statistical properties. Certainly, it is attractive to operate at low frequencies ( $k a \ll 1$, long wavelengths) where there is no modal structure and the echo amplitude PDFs are sharp. 
However, since these features are found in the Rayleigh scattering region, the signal levels would be unacceptably low making the approach impractical. At the other extreme, with very high frequencies ( $k a \gg 1$ ), the acoustic wave is damped inside and around the target reducing the modal structure. This phenomena is illustrated in the upper $k a$ portion of the data in Fig. 5. As $k a$ is increased, the vertical spread in data decreases. Thus it is apparent that the operating frequency of a single-frequency system should be high $(k a \gg 1)$. To further improve the accuracy of the dual-beam technique at these high frequencies, one could sweep the frequency of the system across a relatively narrow band and average the resultant modal oscillations. This approach is being investigated by Zakharia et al. (1989) who is developing a chirp system for application in fisheries acoustics.

Another consideration for work at very high frequencies, is that all echo amplitudes should obey the same statistics (in this case, Rayleigh), allowing one to use the same processing algorithm on all animals. However, because of the great variations present for Rayleigh distributed echoes, many pings must be collected and averaged from the same animal. This requirement poses the greatest practical problem, although a secondary one is the fact that at the higher frequencies, the operating range of the sonar is lower. Since many pings must be collected, the ping rate must be relatively high and the animal must be tracked in order that the postprocessing algorithm "knows" which echoes to average. Depending on the resolution required in the size estimates, at least 10, if not many more echoes, should be averaged per animal in order to accurately size it with a single frequency system. The system would also need to be towed or cast near the animals of interest due to the reduction of operating range at the higher frequencies.

A final source of ambiguity is the possible temperature dependence of TS. Because the density and speed of sound of the animals are so close to that of the surrounding water (each are to within several percent of the corresponding value in water) their values and the resultant TS are potentially very sensitive to variations in temperature. Greenlaw (1977) has shown that for a variation of temperature from $10.5^{\circ} \mathrm{C}$ to $19^{\circ} \mathrm{C}$, the speed of sound contrast, $h$, of preserved euphausiids changes from about 1.0094 to about 1.028 (values taken from Fig. 3 of his article), while the density, $g$, stayed constant at a value of 1.043 (Table $I$ of his article). Assuming the target strength is dominated by the planewave/plane-interface coefficient $R=(g h-1) /(g h+1)$ at these high frequencies (Stanton, 1989b), the target strength would increase by about $2.6 \mathrm{~dB}$ over this temperature range. This phenomenon would strongly influence the accuracy of both single- and multiple-frequency approaches (for example, an error of $2.6 \mathrm{~dB}$ in target strength corresponds to an error of a factor of almost 2 in biomass estimates), although at least relative size frequency distribution is obtainable from the latter system when the temperature is not known. Since there is no parameter to vary in a single-frequency system, the temperature dependence of TS as well as the temperature of the water where the field measurements are made should be determined. We do note that Greenlaw's work involved a limited set of data with preserved animals and there has been no extensive controlled experiments studying the temperature dependence (if any) of the material properties of live organisms. Thus any related concerns at this date are strictly speculative.

Because of the many practical advantages of using a dual-beam system, it is important to choose the optimal sampling scheme that minimizes the disadvantages involved in using just a single frequency. A high enough frequency should be used $(k a \gg 1)$ to reduce the oscillatory behavior of the modal interference pattern while at the same time creating statistical homogeneity of the echo amplitudes. By using a sufficiently high ping rate and an accurate target-tracking algorithm, the mean TS from individual animals can be estimated with far greater precision than has been done in the past.

\section{CONCLUSIONS}

After examining the sound-scattering behavior of a variety of live zooplankton and micronekton, we can draw several conclusions. One of the major conclusions from our experiments is that crustacean zooplankton and micronekton backscatter sound as a function of their volume rather than their cross-sectional area. In addition, the elongate shape of many of these crustaceans can play a major role in determining their sound-scattering properties. For example, the backscatter from such elongate crustaceans appears to be better simulated by cylindrical rather than spherical scattering models. In particular, our work verifies the need for more sophisticated scattering models (e.g., Stanton 1989a, b, submitted) as well as empirical parameterization of such models. While there is convincing evidence in this paper that animals backscatter sound in a manner resembling cylinder models, the predictions based on straight or uniformly bent finite-length cylinder models only approximated the general trends in the data. It is obvious that a more precise shape in the models and much more information on animal morphology and behavior are required to improve upon the accuracy of the model predictions.

As the general behavior of the sound scattering by individual animals becomes better understood, we can improve upon the accuracy of current acoustical techniques for estimating the distribution and abundance of zooplankton and micronekton. New scattering models and improved acoustical techniques, of the types described in this paper, will greatly improve the accuracy of our present-day single- and multiple-frequency systems.

While an understanding of the scattering behavior of live animals has increased through our analysis, much additional work needs to be performed. Sound scattering depends intimately upon the morphology of the animal as well as its size, shape, and orientation. To date, these properties are at best known qualitatively, resulting in speculative values for some of the parameters used in scattering models. More quantitative measurements of these properties must be developed and made in concert with future acoustical measurements to advance our understanding of sound scattering in the ocean. 


\section{ACKNOWLEDGMENTS}

We thank Andy Shedlock for the able assistance he provided during our experimental studies and personnel at the Friday Harbor Laboratories who made every effort to support the logistical aspects of our work. We also thank Bob Eastwood of the Woods Hole Oceanographic Institution for his computer programming and graphical analyses of some of our scattering data. This research was supported by the Oceanic Biology and Ocean Acoustics Programs of the Office of Naval Research Contract Nos. N00014-87-K-007 and N00014-89-J-1729, respectively, and the National Science Foundation Grant No. OCE-8709962. This paper is Contribution No. 7427 of the Woods Hole Oceanographic Institution and No. 2 of the Bioacoustical Oceanography Applications and Theory Center at Cornell University.

\section{APPENDIX: AMPLIFICATION OF SOME FORMULAS USED IN TEXT}

The Rice PDF (Rice, 1954) is presented because our use of $\gamma$ and $\sigma_{\mathrm{bs}}$ will not be found in Rice's paper. Following Clay and Heist (1984), we let $\sigma_{c}$ represent the coherently scattered power and $\sigma_{i}$ represent the average incoherently scattered power (noise), and define the mean backscattering cross section as

$$
\bar{\sigma}_{\mathrm{bs}} \equiv \sigma_{c}+\sigma_{i}
$$

We also define $\gamma$ as the ratio

$$
\gamma \equiv \sigma_{c} / \sigma_{i} \text {. }
$$

The Rice PDF of the echo amplitude $\left(\sqrt{\sigma_{\mathrm{bs}}}\right)$ is

$$
\begin{aligned}
\omega_{R}\left(\sigma_{\mathrm{bs}}^{1 / 2}\right)= & \frac{2 \sigma_{\mathrm{bs}}^{1 / 2}(1+\gamma)}{\overline{\sigma_{\mathrm{bs}}}} \\
& \times \exp \left(-\frac{(1+\gamma) \sigma_{\mathrm{bs}}+\gamma \overline{\sigma_{\mathrm{bs}}}}{\overline{\sigma_{\mathrm{bs}}}}\right) I_{0}(x),
\end{aligned}
$$

where

$$
x \equiv 2 \sigma_{\mathrm{bs}}^{1 / 2}[\gamma(1+\gamma)]^{1 / 2} /\left(\overline{\sigma_{\mathrm{bs}}}\right)^{1 / 2}
$$

and $I_{0}$ is the modified Bessel function (note the difference in our notation versus that of Clay and Heist: $\bar{\sigma}_{\mathrm{bs}}$ in Eq. (A1) of this article refers to the mean backscattering cross section while Clay and Heist present the mean without the bar).

We also provide here the high-pass model equation for the bent cylinder (taken from Stanton, 1989b) and parameter values used in the solutions used in the development of Fig. 5. The high-pass bent cylinder model is given as

$$
\sigma_{\mathrm{bs}}=\frac{\frac{1}{4} L^{2}(k a)^{4} \alpha_{\pi c}^{2} H^{2} G}{1+\left[L^{2}(k a)^{4} \alpha_{\pi c}^{2} H^{2}\right] /\left(\rho_{c} a R^{2} F\right)},
$$

where

$$
\begin{aligned}
& \alpha_{\pi c}=\left(1-g h^{2}\right) / 2 g h^{2}+(1-g) /(1+g), \\
& R=(g h-1) /(g h+1),
\end{aligned}
$$

$g$ is the (density of body material)/(density of surrounding fluid), $h$ is the (compressional speed of sound in body material)/(compressional speed of sound in surrounding fluid), $H=\frac{1}{2}+\frac{1}{2}\left(\rho_{c} / L\right) \sin \left[\left(\rho_{c} / L\right)^{-1}\right], a$ is the cylindrical radius, and $L$ is the arc length of the bent cylinder. The param- eters to all curves involve the following values: $g=1.0357$ and $h=1.0279$ [specific density and speed of sound for live plankton (Foote, 1990)], and for the bent cylinder: $\rho_{c} / L=3$ ( $\rho_{c}=$ radius of curvature of bent cylinder, an estimate) and $L / a=10.5$ (acoustically determined in Stanton, 1989 a). For highpass model \#2, $F=3.0+0.0015(k a)^{4.0}$ and $G=1-0.8 e^{-2.5(k a-2.05)^{2}}$; they were taken from the euphausiid column of Table IV of Stanton (1989b). The highpass model \#1, which was created to fit the truncated modal series solution in Fig. 5, required the following function: $F=5(k a)^{0.4}$ and $G=1-0.87 e^{-2.5(k a-2.0)^{2}}$.

Anderson, N. R., and Zahuranec, B. J. (Eds.) (1977). Oceanic Sound Scattering Prediction (Plenum, New York), 859 pp.

Anderson, V. C. (1950). "Sound scattering from a fluid sphere," J. Acoust. Soc. Am. 22, 426-431.

Clay, C. S., and Medwin, H. (1977). Acoustical Oceanography: Principles and Applications (Wiley-Interscience, New York).

Clay, C. S., and Heist, B. G. (1984). "Acoustic scattering by fish-Acoustic models and a two parameter fit," J. Acoust. Soc. Am. 75, 1077-1083.

Costello, J. H., Pieper, R. E., and Holliday, D. V. (1989). "Comparison of acoustic and pump sampling techniques for the analysis of zooplankton distributions," J. Plankton Res. 11, 703-709.

Ehrenberg, J. E. (1989). "A review of target strength estimation techniques," in Underwater Acoustic Data Processing, edited by Y. T. Chan (Kluwer, Dordrecht), pp. 161-176.

Falk-Petersen, S., and Kristensen, A. (1985). "Acoustic assessment of krill stocks in Ullsfjorden, north Norway," Sarsia 70, 83-90.

Farquhar, G. B. (Ed.) (1971). Proceeding of an International Symposium on Biological Sound Scattering in the Ocean (Maury Center for Ocean Science, Washington, DC).

Foote, K. G., Everson, I., Watkins, J. L., and Bone, D. G. (1990). "Target stengths of Antarctic krill (Euphausia superba) at 38 and $120 \mathrm{kHz}$," J. Acoust. Soc. Am. 87, 16-24.

Greene, C. H., and Wiebe, P. H. (1988). "New developments in bioacoustical oceanography: Application of the dual-beam technique to studies of zooplankton and micronekton ecology," Sea Technol. 29, 27-29.

Greene, C. H., and Wiebe, P. H. (1990). "Bioacoustical oceanography: New tools for zooplankton and micronekton research in the 1990's," Oceanography 3, 12-17.

Greene, C. H., Wiebe, P. H., Burczynski, J., and Youngbluth, M. J. (1988) "Acoustical detection of high-density krill demersal layers in the submarine canyons off Georges Bank," Science 241, 359-361.

Greene, C. H., Wiebe, P. H., and Burczynski, J. (1989a), "Analyzing zooplankton size distributions using high-frequency sound," Limnol. Oceanogr. 34, 129-139.

Greene, C. H., Wiebe, P. H., and Burczynski, J. (1989b). "Analyzing distribution of zooplankton and micronekton using high-frequency, dualbeam acoustics," Progr. Fish. Acoust. 11, 45-54.

Greenlaw, C. F. (1977). "Backscattering spectra of preserved zooplankton," J. Acoust. Soc. Am. 62, 44-52.

Greenlaw, C. F. (1979). "Acoustic estimation of zooplankton populations," Limnol. Oceanogr. 24, 226-242.

Greenlaw, C. F., and Johnson, R. K. (1983). "Multiple-frequency acoustical estimation," Biol. Oceanogr. 2, 227-252.

Holliday, D. V. (1980). "Use of acoustic frequency diversity for marine biological measurements," in Advanced Concepts in Ocean Measurements for Marine Biology, edited by F. P. Diemer, F. J. Vernberg, and D. Z. Mirkes (Univ. South Carolina, Columbia), pp. 423-460.

Holliday, D. V., and Pieper, R. E. (1980). "Volume scattering strengths and zooplankton distributions at acoustic frequencies between 0.5 and 3 MHz," J. Acoust. Soc. Am. 67, 135-146.

Holliday, D. V., Pieper, R. E., and Kleppel. G. S. (1989). "Determination of zooplankton size and distribution with multifrequency acoustic technology," J. Cons. Int. Explor. Mer. 46, 52-61.

Jensen, A. L. (1986). "Functional regression and correlation analysis," Can. J. Fish. Aquat. Sci. 43, 1742-1745.

Johnson, R. K. (1977). “Acoustic estimation of scattering-layer composi- 
tion," J. Acoust. Soc. Am. 61, 375-377.

Kristensen A., and Dalen, J. (1986). "Acoustic estimation of size distribution and abundance of zooplankton," J. Acoust. Soc. Am. 80, 601-611.

Macaulay, M. C. (1978). "Quantitative acoustic assessment of zooplankton standing stock," PhD. thesis, Univ. Washington, Seattle, WA

Pieper, R. E. (1979). "Euphausiid distribution and biomass determined acoustically at $102 \mathrm{kHz}$," Deep-Sea Res. 26, 687-702.

Pieper, R. E., and Holliday, D. V. (1984). "Acoustic measurements of zooplankton distributions in the sea," J. Cons. Int. Explor. Mer 41, 226-238.

Rice, S. O. (1954). "Mathematical analysis of random noise," in Selected Papers on Noise and Stochasic Processes, edited by N. Wax (Dover, New York), pp. 133-294.

Ricker, W. E. (1973). "Linear regression in fishery research," J. Fish. Res. Board Can. 32, 1494-1498.

Richter, K. E. (1985a). "Acoustic scattering at $1.2 \mathrm{MHz}$ from individual zooplankters and copepod populations," Deep-Sea Res. 32, 149-161.

Richter, K. E. (1985b). "Acoustic determination of small-scale distributions of individual zooplankters and zooplankton aggregations," Deep-
Sea Res. 32, 163-182.

Sameoto, D. D. (1976). "Distribution of sound scattering layers caused by euphausiids and their relationship to chlorophyll a concentration in the Gulf of St. Lawrence estuary," J. Fish. Res. Board Can. 33, 681-687.

Stanton, T. K. (1988). "Sound scattering by cylinders of finite length. $I$. Fluid cylinders," J. Acoust. Soc. Am. 83, 55-63.

Stanton, T. K. (1989a). "Sound scattering by cylinders of finite length. III. Deformed cylinders," J. Acoust. Soc. Am. 86, 691-705.

Stanton, T. K. (1989b). "Simple approximate formulas for backscattering of sound by spherical and elongated objects," J. Acoust. Soc. Am. 86, 1499-1510.

Stanton, T. K. (submitted). "Sound scattering by randomly rough elongated objects. I. Means of scattered field," J. Acoust. Soc. Am.

Stanton, T. K. (in preparation). "Sound scattering by randomly rough elongated objects. II. Fluctuations of scattered field," J. Acoust. Soc. Am.

Zakharia, M., Corgiatti, J. P., Joly, F., and Person, R. (1989). "Wide-band sounder for fisheries," Proc. Inst. Acoust. 11(8), 274-281. 